\title{
FINITELY EMBEDDED COMMUTATIVE RINGS
}

\author{
CARL FAITH
}

(Communicated by Louis J. Ratliff, Jr.)

\begin{abstract}
A theorem of Ginn and Moss [G-M] states that a right finitely embedded ( $=$ with finite essential right socle) two-sided Noetherian ring is Artinian. An example of Schelter and Small [S-S] can be applied to show that the theorem fails for right finitely embedded rings with the ascending chain conditions on right and left annihilators. We show here, however, that finitely embedded commutative rings with the acc on annihilators $(=\operatorname{acc} \perp)$ are Artinian. The proof uses the author's characterization in [F1] of acc $\perp$ rings, and the Levitzki [L] and Herstein-Small [H-S] theorem on the nilpotency of nil ideals in 2-sided acc $\perp$ rings. A corollary is a result of Shizhong [Sh] that shows commutative subdirectly irreducible acc $\perp$ rings are $\mathrm{QF}$.
\end{abstract}

We first prove the main theorem.

1. Theorem. A finitely embedded commutative ring $R$ with acc $\perp$ is Artinian. Proof. Let $S=V_{1} \oplus \cdots \oplus V_{n}$ be the socle of $R$. Thus, $V_{i}$ is a minimal ideal of $R$, and the annihilator $V_{i}^{\perp}$ of $V_{i}$ in $R$ is a maximal ideal $M_{i}$, where $R / M_{i} \approx V_{i}, i=1, \ldots, n$. It follows that $S$ has annihilator

$$
J=S^{\perp}=\bigcap_{i=1}^{n} M_{i} .
$$

Since the singular ideal $Z$ of a ring $R$ is the set of those $x \in R$ with $x^{\perp}$ an essential right ideal, it follows that $x V_{i}=0, i=1, \ldots, n$ for each $x \in Z$, that is, that

$$
Z=S^{\perp}=J
$$

By the result of Levitzki-Herstein-Small, $Z$ is nilpotent, since acc $\perp$ implies that $Z$ is nil (see, e.g., $[F]$ ).

It follows that $J$ is the Jacobson radical of $R$, since the Jacobson radical contains every nil ideal and is contained in every maximal ideal. Since $J=$ $\bigcap_{i=1}^{n} M_{i}$ is a finite intersection of maximal ideals, $R / J$ is semisimple Artinian, so every $R / J$-module is semisimple.

Received by the editors April 3, 1990.

1980 Mathematics Subject Classification (1985 Revision). Primary 13C05, 13E05, 13E10, 13E15; Secondary 16A34, 16A35, 16A36. 
We next prove that $R$ has a composition series. Let $n$ be the index of nilpotency of $J$, and consider the socle series

$$
S=S_{1} \subseteq S_{2} \subseteq \cdots \subseteq S_{n-1} \subset R=S_{n},
$$

where $S_{i}=\left(J^{i}\right)^{\perp}$. Since $S_{i+1} J \subseteq S_{i}$, then $S_{i+1} / S_{i}$ is a semisimple module, $\forall i$. By a theorem of $[F 1]$, a ring with (right) acc $\perp$ has the property that every (left) annihilator ideal $I$ is finitely annihilated, say $I=L_{1}^{\perp}$, where $L_{1}=$ $\left(x_{1}, \ldots, x_{n}\right)$. This implies, however, that the factor module $R / I$ embeds in the free $R$-module $R^{n}$ under a map

$$
r+I \mapsto\left(x_{1} r, \ldots, x_{n} r\right) \in R^{n} \quad \forall r \in R .
$$

In particular, since $R$ is finitely embedded, then $R^{n}$, whence $R / I$, is finitely embedded; hence, each $R / S_{i}$ has finite socle. But $\operatorname{soc}\left(R / S_{i}\right) \supseteq S_{i+1} / S_{i}$ since $S_{i+1} / S_{i}$ is semisimple, proving that $S_{i+1} / S_{i}$ has finite (Jordan-Holder) length. Since $S_{n-1} \supseteq J$, and since $R / J$ has finite length, then $R / S_{n-1}$ and every $S_{i+1} / S_{i}$ has finite length. This prove that $R$ is Artinian.

A semisimple module $M$ is square-free if $M$ contains no submodules $\approx V^{2}$ where $V$ is simple; i.e., iff $M=\bigoplus_{i} V_{i}$ for nonisomorphic simple modules $V_{i}$.

2. Corollary. A commutative ring $R$ is QF iff $R$ is a finitely square-free embedded acc $\perp$ ring.

Proof. Square-free socle is a well-known characterization of QF rings among commutative Artinian rings. If $R$ is Artinian, then $R=\prod_{i=1}^{n} R_{i}$ where $R_{i}$ is local. By classic ideal theory, any Noetherian subdirectly irreducible (SDI) ring is $\mathrm{QF}$ (e.g. see [V]), and conversely, any QF local ring is SDI. The corollary follows from this and Theorem 1.

3. Corollary (Shizhong [Sh]). $A$ SDI commutative ring $R$ is QF iff $R$ is an acc $\perp$ ring.

4. Example. A commutative semiprimary ring with acc $\perp$ need not be Noetherian. Let $A=k\left\langle x_{1}, \ldots, x_{n}, \ldots\right\rangle$ be the power series ring over a field $k$ in infinitely many variables, and let $J$ be the unique maximal ideal. Then $R=A / J^{2}$ is a semiprimary ring with infinite socle, and $J / J^{2}$ is the only nontrivial annihilator of $R / J^{2}$. Thus, $R$ has acc $\perp$ but is not Noetherian.

5. Example. Consider the ring $R$ of $[\mathrm{S}-\mathrm{S}]$ that is right Artinian but with maximal right quotient ring $Q=Q_{\max }^{r}(R)$ that is not right Artinian. It can be shown (see, e.g., [F3, Part I]) that $Q$ is a semiprimary right Goldie ring with (2-sided) acc $\perp$. Since $Q$ is semiprimary, it has essential right socle $S$, and since it is right Goldie, $S$ has finite length. Thus, $Q$ is the desired example: $Q$ has 2-sided acc $\perp$, finitely embedded yet not right Artinian.

6. Remarks on the literature. In [C], Camillo proves that a commutative SDI acc $\perp$ ring is perfect. In [F1] it is proved that a left perfect right acc $\perp$ ring is semiprimary. Theorem 1 then implies that a commutative perfect ring is Artinian iff $R$ is Goldie. 


\section{REFERENCES}

[C] V. Camillo, Commutative rings whose quotients are Goldie, Glasgow Math. J. 16 (1975), 32-33.

[F] C. Faith, Lectures on injective modules and quotient rings, Lecture Notes in Math., vol. 49, Springer-Verlag, Berlin, 1967.

[F1] __ Rings with ascending condition on annihilators, Nagoya Math. J. 27 (1966), 179-191.

[F2] _ Algebra II: ring theory, Springer-Verlag, Berlin, 1976.

[F3] _ Injective modules over Levitzki rings, Part I of Injective modules and injective quotient rings, Lecture Notes in Pure and Appl. Math., vol. 72, Marcel Dekker, New York, 1982.

[F4] _ Annihilators, associated prime ideals, and Kasch-McCoy quotient rings of commutative rings, Robert Warfield Memorial Issue, Comm. Algebra (to appear).

[H-S] I. N. Herstein and L. Small, Nil rings satisfying certain chain conditions, Canad. J. Math. 16 (1964), 771-776.

[L] J. Levitzki, On nil subrings, Israel Math. J. 1 (1963), 215-216.

[S-S] W. Schelter and L. Small, Some pathological rings of quotients, J. London Math. Soc. 14 (1976), 200-202.

[Sh] P. Shizhong, Commutative subdirectly irreducible rings with the acc on annihilators are QF, Comm. Algebra (to appear).

[V] P. Vámos, Classical rings, J. Algebra 34 (1975), 114-129.

Department of Mathematics, Rutgers University, New Brunswick, New Jersey 08903 AFFILIATION

Current address: 199 Longview Drive, Princeton, New Jersey 08540 